\title{
Initial multicenter experience with a new High-Density coloring module: impact for complex atrial arrhythmias interpretation
}

\author{
Grégoire Massoullié ${ }^{1}$, Ghassan Moubarak ${ }^{2}$, Olivier Thomas ${ }^{2}$, Alexis Mechulan ${ }^{3}$, Antoine \\ Da Costa ${ }^{4}$, Vincent Roger ${ }^{5}$, Julio Justo ${ }^{6}$, Romain Eschalier ${ }^{7}$, john silberbauer ${ }^{6}$, and Marius \\ Andronache $^{1}$ \\ ${ }^{1}$ Centre Hospitalier Universitaire de Clermont-Ferrand \\ ${ }^{2}$ Centre Médico-chirurgical Ambroise Paré \\ ${ }^{3}$ Ramsay Général de Santé \\ ${ }^{4}$ Centre Hospitalier Universitaire de Saint-Etienne \\ ${ }^{5}$ Biosense Webster Inc \\ ${ }^{6}$ Brighton and Sussex University Hospitals NHS Trust \\ ${ }^{7}$ Clermont Université
}

April 27, 2020

\begin{abstract}
Background: High-density automated mapping of complex atrial tachycardias (ATs) requires accurate assessment of activation maps. A new local activation display module (HD Coloring, Biosense Webster@) provides higher map resolution, a better delineation of potential block reducing color interpolation, and a new propagation display. We evaluated the accuracy of a dedicated local activation display compared to standard algorithm. Methods: High density maps from 10 organized atrial tachycardias were collected with a multipolar catheter and were displayed with standard activation or HD Coloring. Six blinded expert operators retrospectively analyzed activation maps and were asked to define (1) the tachycardia mechanism, (2) ablation target, and (3) level of difficulty to interpret those maps. Results: Using HD Coloring, operators were able to reach a correct diagnosis in $93 \%$ vs. $63 \%, \mathrm{p}<0.05$ compared to standard activation maps. Time to diagnosis was shorter $1.9 \pm 1.0$ min vs. $3.9 \pm 2.1 \mathrm{~min}, \mathrm{p}<0.05$. Confidence level would have allowed ablation without necessity for entrainment maneuvers in $87 \%$ vs. $53 \%, \mathrm{p}<0.05$. Operators would have needed to remap or proceed with multiple entrainments in $3 \%$ vs. $13 \%$ of cases, $\mathrm{p}<0.05$. Finally, ablation strategy was more accurately identified in $97 \%$ vs. $67 \%, \mathrm{p}<0.05$. Conclusion: Activation mapping with the new HD Coloring Module allowed a more accurate, reliable, and faster interpretation of complex ATs mechanisms compared to standard activation maps.
\end{abstract}

\section{Hosted file}

HDJCEV4. docx available at https : //authorea.com/users/315702/articles/446161-initial-multicenterexperience-with-a-new-high-density-coloring-module-impact-for-complex-atrial-arrhythmiasinterpretation 


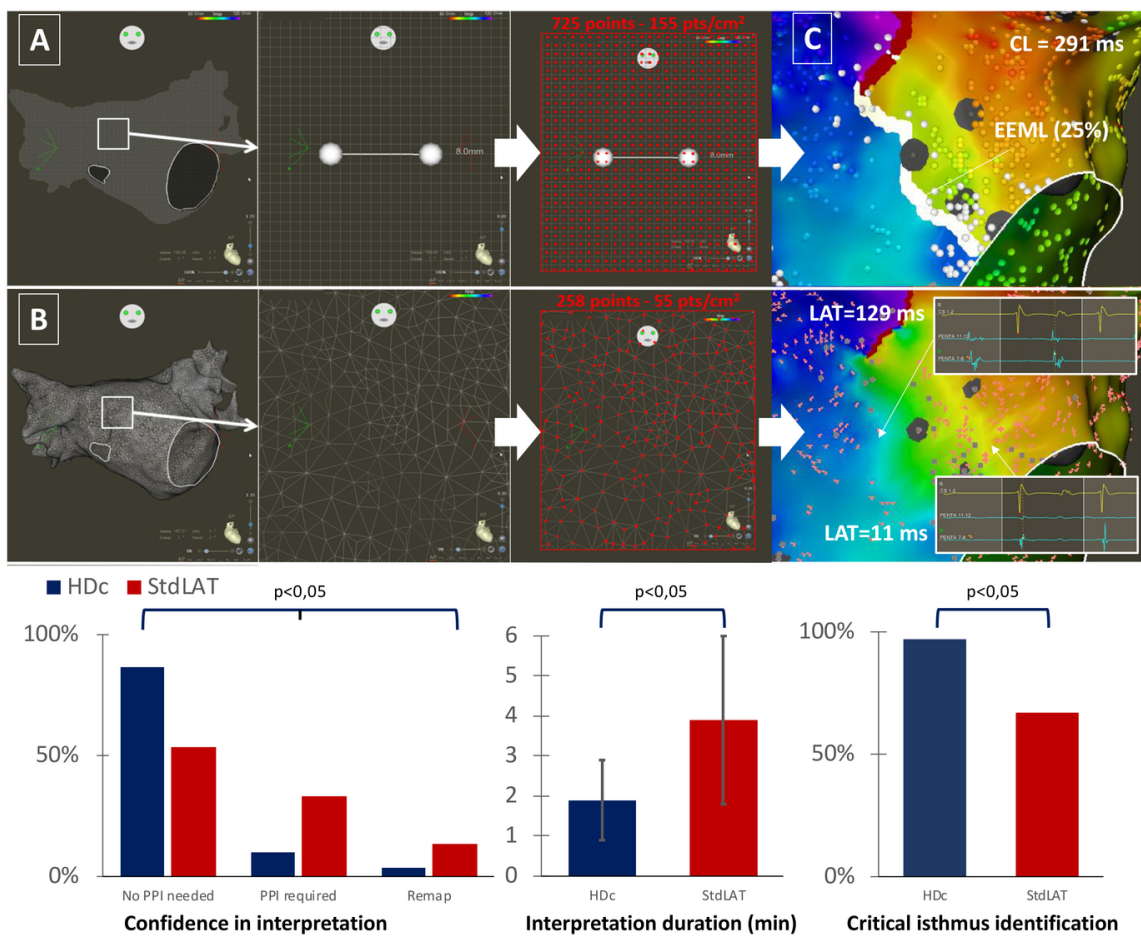




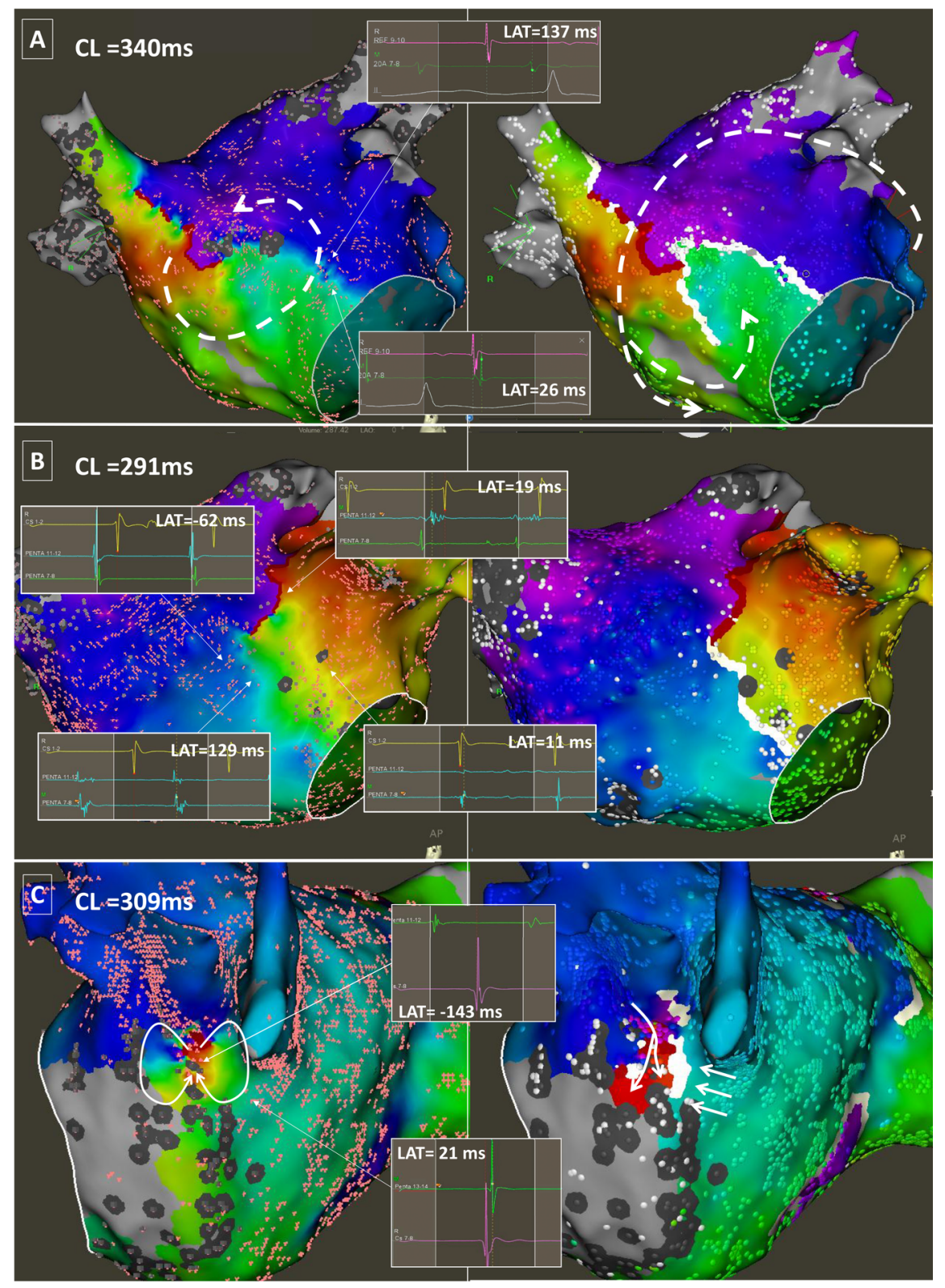

\section{Hosted file}

Video 1.avi available at https://authorea.com/users/315702/articles/446161-initial-multicenterexperience-with-a-new-high-density-coloring-module-impact-for-complex-atrial-arrhythmiasinterpretation 